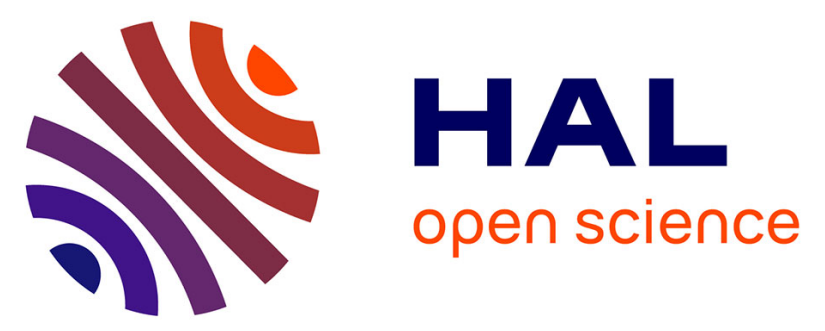

\title{
Effets uro-néphrologiques des produits utilisés par les usagers de drogues : revue de la littérature et enquête pharmaco-épidémiologique en France et dans la région de Marseille
}

Marion Gully, Élisabeth Frauger, Michel Spadari, Liselotte Pochard, Vanessa

Pauly, Fanny Romain, Bertrand Gondouin, Marion Sallée, Julie

Moussi-Francès, Stéphane Burtey, et al.

\section{To cite this version:}

Marion Gully, Élisabeth Frauger, Michel Spadari, Liselotte Pochard, Vanessa Pauly, et al.. Effets uro-néphrologiques des produits utilisés par les usagers de drogues : revue de la littérature et enquête pharmaco-épidémiologique en France et dans la région de Marseille. Néphrologie \& Thérapeutique, 2017, 13 (6), pp.429-438. 10.1016/j.nephro.2017.01.024 . hal-01629473

HAL Id: hal-01629473

https://hal-amu.archives-ouvertes.fr/hal-01629473

Submitted on 9 Dec 2017

HAL is a multi-disciplinary open access archive for the deposit and dissemination of scientific research documents, whether they are published or not. The documents may come from teaching and research institutions in France or abroad, or from public or private research centers.
L'archive ouverte pluridisciplinaire HAL, est destinée au dépôt et à la diffusion de documents scientifiques de niveau recherche, publiés ou non, émanant des établissements d'enseignement et de recherche français ou étrangers, des laboratoires publics ou privés. 


\title{
Effets uro-néphrologiques des produits utilisés par les usagers de drogues : revue de la littérature et enquête pharmaco-épidémiologique en France et dans la région de Marseille
}

\author{
Uro- and nephrotoxic effects of drugs of abuse: Literature review and pharmaco-epidemiological \\ survey in France and in the Marseille area
}

\author{
Marion Gully ${ }^{\mathrm{a}}$, Élisabeth Frauger ${ }^{\mathrm{b}}$, Michel Spadari ${ }^{\mathrm{b}}$, Liselotte Pochard ${ }^{\mathrm{b}}$, Vanessa Pauly ${ }^{\mathrm{c}}$, \\ Fanny Romain ${ }^{c}$, Bertrand Gondouin ${ }^{a}$, Marion Sallée ${ }^{a}$, Julie Moussi-Frances ${ }^{a}$, \\ Stéphane Burtey ${ }^{\mathrm{a}}$, Bertrand Dussol ${ }^{\mathrm{a}}$, Laurent Daniel $^{\mathrm{d}}$, Joëlle Micallef ${ }^{\mathrm{b}}$, \\ Noémie Jourde-Chiche ${ }^{\mathrm{a}, *}$ \\ ${ }^{a}$ Centre de néphrologie et transplantation rénale, hôpital de la Conception, Aix-Marseille université, AP-HM, 147, boulevard Baille, 13005 Marseille, France \\ ${ }^{\mathrm{b}}$ Service de pharmacologie, CEIP-addictovigilance PACA-Corse, Aix-Marseille université, CHU de la Timone, AP-HM, 264, rue Saint-Pierre, 13385 Marseille, \\ France \\ ' Service d'information médicale, hôpital de la Conception, Aix-Marseille université, AP-HM, 147, boulevard Baille, 13005 Marseille, France \\ d Département d'anatomopathologie, Aix-Marseille université, CHU de la Timone, AP-HM, 264, rue Saint-Pierre, 13385 Marseille, France
}

Mots clés :

Glomérulonéphrite

Insuffisance rénale aiguë

Insuffisance rénale chronique

Substances psychoactives

Usager de drogue

Vascularite

\section{R É S U M É}

L'abus de substances psychoactives (SPA) s'est diversifié ces dernières années, tant au niveau des populations concernées que des produits consommés. Si la dépendance et les troubles psychiatriques liés à la consommation de ces substances sont généralement connus, les complications somatiques, en particulier uro-néphrologiques, le sont moins. Nous proposons tout d'abord dans cette revue un état des lieux des SPA consommées par les usagers de drogue en France, à partir des données de l'étude nationale pharmaco-épidémiologique OPPIDUM (Observation des produits psychotropes illicites ou détournés de leur utilisation médicamenteuse). Parmi les 5003 patients interrogés, 84 \% étaient consommateurs d'au moins une SPA médicamenteuse, avec des indicateurs d'abus dans $28 \%$ des cas, et plus de la moitié avaient consommé une SPA non médicamenteuse (principalement du cannabis) la semaine précédente. Nous décrivons ensuite les complications urologiques et néphrologiques des principales SPA, telles que l'héroïne, la cocaïne, le cannabis, l'ecstasy, le LSD, les amphétamines, les nouveaux produits de synthèse, la kétamine et les médicaments de substitution aux opiacés. Nous présentons enfin une enquête pharmaco-épidémiologique concernant les patients hospitalisés en néphrologie adulte au CHU de Marseille pour des complications liées à l'abus de SPA. Entre 2000 et 2015, 22 patients âgés de 18 à 57 ans ont été hospitalisés pour des complications néphrologiques des SPA, telles que glomérulonéphrite aiguë, hyalinose segmentaire et focale, insuffisance rénale aiguë ou chronique. Les complications somatiques liées à l'usage des SPA participent à leur dangerosité et peuvent avoir une valeur d'alerte. Il est important que les professionnels de santé signalent au centre d'addictovigilance de leur territoire les complications sanitaires liées à l'usage des SPA, afin d'optimiser l'information donnée aux patients et aux professionnels de santé et d'adapter des programmes de prévention et de réduction des risques.

\footnotetext{
* Auteur correspondant.

Adresse e-mail : noemie.jourde@ap-hm.fr (N. Jourde-Chiche).
} 
Keywords:

Acute kidney injury

Chronic kidney disease

Drug abuse

Glomerulonephritis

Vasculitis

Agreatdiversification of drugs of abuse has been observed in recentyears, bothin the populationsusing them and in the types of drugs. Although dependency and psychiatric disorders associated with the abuse of these substances is well known, somatic complications, uro-nephrotoxicity in particular, are less recognized. We propose hereanoverview of the productsused by drugsabusersinFrance, through theanalysis of thenational pharmaco-epidemiological study Observationdesproduitspsychotropes illicitesoudétournésdeleurutilisation médicamenteuse (OPPIDUM). Among the 5003 patients who participated in this survey, $84 \%$ were on prescribed psychoactive substances, with indicators of abuse in $28 \%$ of cases; more than half of thesepatients had also been using drugs of abuse (mainly cannabis) in the previous week. We then describe the main urological and renal toxicities of these drugs, in particular of heroin, cocaine, cannabis, ecstasy, LSD, amphetamine, new designer drugs, ketamine and opiate substitution treatment. We finally present a pharmaco-epidemiological survey of patients hospitalized for drugs complications in nephrology at the university hospital of Marseille. Between2000and2015, 22 patients aged 18 to57years werehospitalized for renal adverse effects of drugs of abuse, such as glomerulonephritides, focal segmental glomerulosclerosis, acute kidney injury or chronic kidney disease. The somatic complications of drugs participate in their dangerousness and should be a red flag. They should be systematically reported to the addictovigilance nationalnetworktoallow the improvement ofinformationgiventothepatientsand themedicalcommunity, and to adapt the prevention and risk reduction policies.
\end{abstract}

\section{Abréviations}

\begin{tabular}{|c|c|}
\hline AMM & autorisation de mise sur le marché \\
\hline ANCA & anticorps anti-cytoplasme des polynucléaires neutrophiles \\
\hline ANSM & agence nationale de sécurité du médicament \\
\hline Elsa & équipe de liaison de soins en addictologie \\
\hline GNA & glomérulonéphrite aiguë \\
\hline GNMP & glomérulonéphrite membrano-proliférative \\
\hline HSF & hyalinose segmentaire et focale \\
\hline IRA & insuffisance rénale aiguë \\
\hline IRC & insuffisance rénale chronique \\
\hline LSD & diéthylamide d'acide lysergique \\
\hline MAT & microangiopathie thrombotique \\
\hline MDMA & 3,4-méthylène-dioxy-n-méthyl-amphétamine \\
\hline Mildeca & $\begin{array}{l}\text { mission interministérielle de lutte contre les drogues et } \\
\text { les conduites addictives }\end{array}$ \\
\hline NPS & nouveaux produits de synthèse \\
\hline NTA & nécrose tubulaire aiguë \\
\hline OPPIDUM & $\begin{array}{l}\text { observation des produits psychotropes illicites ou } \\
\text { détournés de leur utilisation médicamenteuse }\end{array}$ \\
\hline PAN & périartérite noueuse \\
\hline SPA & substance psychoactive \\
\hline SINTES & système d'identification national des toxiques et substances \\
\hline TSO & traitement de substitution aux opiacés \\
\hline
\end{tabular}

\section{Introduction}

L'abus de substances psychoactives (SPA) s'est diversifié ces dernières années, tant au niveau des populations concernées (usagers de drogues, sujets jeunes, patients douloureux. ..) que des produits consommés et de leurs modalités de consommation. Parmi les SPA pouvant faire l'objet d'abus, il y a bien évidemment les médicaments psychoactifs (opiacés, benzodiazépines et apparentés, anesthésiques, psychostimulants...), mais également des SPA non médicamenteuses (cannabis, héroïne, cocaïne, LSD, amphétamines/speed, MDMA/ecstasy, plantes, champignons, nouveaux produits de synthèse [NPS]...).
Si la dépendance et les troubles psychiatriques liés à la consommation des SPA sont généralement connus, les complications somatiques le sont moins [1]. Ces complications peuvent être liées aux propriétés pharmacologiques des substances ellesmêmes, à leur mode d'administration, ou à la présence de produits de coupe (comme le lévamisole pour la cocaïne).

Ainsi, des complications cardiovasculaires ont été observées chez les consommateurs de cannabis [2], de cannabinoïdes de synthèse [3,4], et de cocaïne [5]. De même, des complications infectieuses peuvent survenir suite à l'injection de produits [6].

Si les effets uro-néphrologiques secondaires à l'héroïne et la cocaïne sont bien connus, l'extension du panel des substances utilisées, y compris en France, avec l'arrivée massive des NPS, nous incite à proposer une mise au point des complications uronéphrologiques rapportées après consommation de SPA. Durant la dernière décennie, les NPS ont en effet été utilisés de façon croissante, pour contourner la législation (euphorisants légaux ou legal highs) et/ou compenser la moindre disponibilité ou qualité des drogues dites classiques, avec une accessibilité par Internet [7]. Les NPS correspondent le plus souvent à des analogues ou dérivés de médicaments ou drogues existants, dont les structures chimiques ont été modifiées à des degrés variables pour un effet plus puissant et plus sélectif, à de plus faibles concentrations. Ces nouvelles drogues sont apparentées à de nombreuses classes chimiques (phénéthylamines, tryptamines, pipérazines, cathinones, cannabinoïdes. . .) regroupant des sousclasses incluant chacune un grand nombre de représentants [8]. Chaque année, de nouvelles molécules sont découvertes et représentent une grande part des consommations de SPA chez les jeunes âgés de 17-18 ans, notamment aux États-Unis [8]. Pourtant, des effets indésirables sont retrouvés chez $70 \%$ des consommateurs environ, dont $5 \%$ conduisent à une hospitalisation [8].

En France, la Mission interministérielle de lutte contre les drogues et les conduites addictives (Mildeca) a publié en septembre 2016 un guide sur les NPS destiné aux services d'urgences, visant à accompagner la prise en charge diagnostique et thérapeutique des patients suspects d'intoxication à un NPS (http://www.drogues.gouv.fr/sites/drogues.gouv.fr/files/atoms/ files/brochure_elsa_nsp.pdf). 
Notre travail comprend trois parties :

- un état des lieux des SPA consommées par les usagers de drogues en France, en caractérisant les modalités de consommation à partir des données de l'étude nationale pharmaco-épidémiologique Observation des produits psychotropes illicites ou détournés de leur utilisation médicamenteuse (OPPIDUM) ;

- une description des différentes atteintes uro-néphrologiques liées à l'utilisation de SPA et de leurs mécanismes à partir des données de la littérature ;

- une enquête réalisée au CHU de Marseille sur les hospitalisations en néphrologie pour des complications en lien avec l'utilisation de SPA, à partir des données de codage des séjours hospitaliers (PMSI) et du logiciel de prescription informatisée.

\section{Matériel et méthodes}

\subsection{Addictovigilance et enquête OPPIDUM}

Sur les recommandations de l'Organisation mondiale de la santé (OMS), un réseau national d'addictovigilance a été créé en 1990, sous l'impulsion de l'Agence nationale de sécurité du médicament (ANSM) afin de surveiller l'abus, l'usage détourné et la pharmacodépendance liés à la prise de SPA (hors alcool et tabac). Les 13 centres d'addictovigilance ont au sein de leur territoire les missions suivantes :

- informer et former les professionnels de santé sur les SPA à risque d'abus ;

- détecter, collecter, évaluer, caractériser et analyser les signaux relatifs à l'abus, la pharmacodépendance et au détournement de toutes les SPA.

Cette vigilance sanitaire s'appuie notamment sur les notifications spontanées des professionnels de santé, au même titre que la pharmacovigilance. Compte tenu du champ exploré et des comportements liés à la consommation des SPA (nomadisme médical, comportement de dissimulation, caractère illégal), des dispositifs de surveillance pharmaco-épidémiologique ont été mis en place afin de compléter les données provenant des notifications spontanées. Le dispositif de surveillance OPPIDUM, étude pharmaco-épidémiologique transversale annuelle nationale existant depuis 1995, permet ainsi de recueillir, grâce à un réseau de structures spécialisées dans la prise en charge des addictions, des informations sur les modalités d'usage des SPA par les patients suivis pour un abus, une dépendance, ou sous traitement de substitution aux opiacés (TSO).

\subsection{Revue de la littérature}

Une recherche a été réalisée sur PubMed à partir des noms des SPA identifiées dans l'enquête OPPIDUM, ainsi que du terme générique drug abuse, et des atteintes uro-néphrologiques suivantes : urinary tract, acute kidney injury, chronic kidney disease, vasculitis, glomerulonephritis.

\subsection{Enquête en néphrologie au CHU de Marseille}

Nous avons réalisé une enquête rétrospective à partir des données du PMSI concernant les patients hospitalisés en néphrologie adulte au CHU de Marseille afin d'évaluer le nombre d'hospitalisations en lien avec la consommation de SPA et les diagnostics retenus.

Parmi les patients hospitalisés de 2000 à 2015 dans le service de néphrologie adulte, ont été sélectionnés, dans un premier temps, les séjours dont l'un des codes diagnostiques (diagnostic principal ou associé) saisis dans le PMSI était en rapport avec la consommation de SPA ou la survenue d'une néphropathie toxique (Tableau 1), ainsi que les patients pour lesquels avait été prescrit un TSO (buprénorphine ou méthadone).

À partir d'un examen plus approfondi des dossiers médicaux, nous avons ensuite exclu les patients ayant une atteinte rénale secondaire à un toxique non SPA, dont l'atteinte rénale n'était pas en lien avec la consommation de SPA, ou dont l'atteinte était secondaire à un surdosage involontaire dans un cadre thérapeutique.

\section{Résultats}

4.1. État des lieux des SPA consommées en France, et résultats de l'étude OPPIDUM 2015

Les principales SPA consommées en France dans le cadre d'un abus/détournement, leur classification, leurs modes de consommation et leurs noms usuels, sont résumés dans le Tableau 2.

Durant l'enquête OPPIDUM d'octobre 2015, 5003 patients ont été inclus. Ils ont décrit la consommation de 10159 SPA la semaine précédant l'entretien. L'âge moyen des sujets était de $36 \pm 10$ ans et

\section{Tableau 1}

Codes CIM-10 retenus pour la sélection des séjours hospitaliers avant analyse des dossiers médicaux.

\begin{tabular}{|c|c|}
\hline Code & Signification \\
\hline F11 & Troubles mentaux et du comportement liés à l'utilisation d'opiacés \\
\hline F12 & Troubles mentaux et du comportement liés à l'utilisation de dérivés du cannabis \\
\hline F14 & Troubles mentaux et du comportement liés à l'utilisation de cocaïne \\
\hline F19 & Troubles mentaux et du comportement liés à l'utilisation de drogues multiples et troubles liés à l'utilisation d'autres SPA \\
\hline F16 & Troubles mentaux et du comportement liés à l'utilisation d'hallucinogènes \\
\hline T40 & Intoxication par narcotiques et psychodysleptiques \\
\hline T41 & Intoxication par anesthésiques et gaz thérapeutiques \\
\hline T620 & Champignons ingérés \\
\hline T622 & Autres parties de plantes ingérées \\
\hline T52 & Effet toxique de solvants organiques \\
\hline T53 & Effet toxique de dérivés halogénés d'hydrocarbures aliphatiques et aromatiques \\
\hline T387 & Androgènes et autres anabolisants \\
\hline N142 & Néphropathie due à un médicament ou une substance biologique, sans précision \\
\hline N144 & Néphropathie toxique, non classée ailleurs \\
\hline T40 & Intoxication par narcotiques et psychodysleptiques (hallucinogènes) \\
\hline X62 & Auto-intoxication par des narcotiques et psychodysleptiques et exposition à ces produits, non classés ailleurs \\
\hline F15 & Troubles mentaux et du comportement liés à l'utilisation d'autres stimulants \\
\hline F18 & Troubles mentaux et du comportement liés à une intoxication aiguë aux solvants \\
\hline
\end{tabular}

SPA : substance psychoactive. 
Tableau 2

Principales substances psychoactives consommées dans le cadre d'un abus/détournement.

\begin{tabular}{|c|c|c|c|}
\hline Produit/famille & Exemples & Principaux modes de prise & Exemples de noms de " rue " \\
\hline \multicolumn{4}{|l|}{ Opiacés } \\
\hline \multirow[t]{2}{*}{$\begin{array}{l}\text { Médicaments de substitution aux } \\
\text { opiacés }\end{array}$} & Buprénorphine haut dosage & $\begin{array}{l}\text { Sublingual } \\
\text { IV, sniffé }\end{array}$ & Sub, subu \\
\hline & Méthadone & Oral, IV et sniffé (rare) & Métha \\
\hline \multirow[t]{3}{*}{ Médicaments antalgiques } & Codéine & Oral & \\
\hline & Tramadol & Oral & \\
\hline & Sulfate de morphine & Oral, IV & \\
\hline Héroïne & & Sniffée, IV, fumée & Came, méca, brown sugar, héro \\
\hline \multirow[t]{2}{*}{ Benzodiazépines et apparentés } & Clonazépam & Oral, IV (rare) & \\
\hline & Zolpidem & Oral, IV (rare) & \\
\hline \multirow[t]{2}{*}{ Arylcyclohexilamines } & Kétamine & Orale, sniffée, IV & K, spécial K, kéta \\
\hline & Méthoxetamine & Orale, sniffée & MXE, légal kétamine \\
\hline \multirow[t]{2}{*}{ Cocaine } & & Sniffée, IV & $\begin{array}{l}\text { Coke, coco, } \mathrm{CC} / \text { produit de coupe comme } \\
\text { le lévamisole }\end{array}$ \\
\hline & & Fumée (crack, freebase) & Caillou \\
\hline \multicolumn{4}{|l|}{ Cannabinoïdes } \\
\hline Cannabis sativa & & $\begin{array}{l}\text { Fumé (herbe, résine) } \\
\text { Oral (spacecake) }\end{array}$ & Shit, joint, beuh, weed \\
\hline Cannabinoïdes de synthèse & $\begin{array}{l}\text { JWH-xxx, CP-xxx, HU-xxx, UR-144, AB- } \\
\text { FUBINANA, AB-PINACA, AB-CHMINACA }\end{array}$ & Fumés, vapotage & $\begin{array}{l}\text { Sachets de plantes à fumer portant des } \\
\text { noms divers tels spice, yuccatan, chill, } \\
\text { K2, black mamba }\end{array}$ \\
\hline \multirow[t]{3}{*}{ Amphétamines et dérivés } & Amphétamine & Oral, sniffé, IV, parachute & Speed, crystal, meth, ice \\
\hline & $\begin{array}{l}\text { Méthylène-Dioxy-Métamphétamine } \\
\text { (MDMA) }\end{array}$ & Oral, sniffé, parachute & Ecstasy, Taz, XTC, exta \\
\hline & Méthylphénidate & Oral, IV, sniffé & \\
\hline Cathinones & $\begin{array}{l}\text { Méphédrone (4-MMC), MDPV, 3-MMC, } \\
\text { 4-MEC, Alpha-PVP }\end{array}$ & Sniffé, IV & $\begin{array}{l}\text { Miaou miaou, NRG, sel de bain, engrais } \\
\text { pour plante }\end{array}$ \\
\hline Tryptamines & LSD & Oral, sublingual & Trip, acide \\
\hline Plantes hallucinogènes & Datura & Oral (infusion, décoction) & \\
\hline Champignons hallucinogènes & Psilocybes & Oral & Psilo \\
\hline \multicolumn{4}{|l|}{ Substances volatiles } \\
\hline Solvants & GBL (précurseur du GHB) & Sniffé, oral & \\
\hline Bombes aérosol & & Sniffé & Déo \\
\hline Poppers & Nitrite de propyle & Sniffé, oral (rare) & Sex line, rush, jungle juice \\
\hline
\end{tabular}

IV : intraveineuse.

78 \% étaient des hommes. Parmi les sujets inclus, $91 \%$ présentaient une dépendance tabagique et $23 \%$ une dépendance alcoolique ; $16 \%$ avaient consommé au moins une SPA par voie nasale, $8 \%$ par voie intraveineuse (IV) et 7 \% par voie inhalée (hors cannabis).

Parmi les sujets, 84 \% étaient consommateurs d'au moins une SPA médicamenteuse (principalement TSO, benzodiazépines et apparentés, antipsychotiques, antidépresseurs et opiacés). Il existait des indicateurs d'abus ou de détournement de ces SPA médicamenteuses chez 28 \% d'entre eux.

Beaucoup étaient également consommateurs de SPA non médicamenteuses : $43 \%$ de cannabis, $11 \%$ d'héroïne, $13 \%$ de cocaïne, 3 \% de drogues de synthèse (ecstasy, amphétamines/ speed, LSD et NPS) et 0,1\% de plantes et champignons. Les voies d'administration de ces SPA non médicamenteuses chez les sujets de l'enquête OPPIDUM sont indiquées sur la Fig. 1.

\subsection{Revue de la littérature sur les effets uro-néphrologiques liés à l'utilisation de SPA}

Les atteintes uro-néphrologiques abordées dans cette partie sont résumées dans le Tableau 3.

\subsubsection{Atteintes urologiques : kétamine, et amphétamines et dérivés}

La consommation d'amphétamines à forte dose, en particulier d'ecstasy, peut être responsable d'une rétention aiguë d'urine [9]. Les amphétamines sont en effet des agonistes alphaadrénergiques, dont les récepteurs sont situés essentiellement au niveau des muscles lisses et ont un rôle constricteur (notamment responsable sur le plan hémodynamique de l'élévation de la pression artérielle). Au niveau du trigone et du col vésical, la fixation d'agonistes adrénergiques induit la contraction des cellules musculaires lisses et la fermeture du col vésical aboutissant à une rétention aiguë d'urine.

L'abus chronique de kétamine peut également conduire à des complications urologiques. Ce traitement est utilisé en anesthésie (AMM) ou dans le cas de douleurs chroniques rebelles (soins palliatifs selon la recommandation ANSM 2010). Son utilisation est parfois détournée par certains usagers recherchant des effets euphorisants ou hallucinogènes. Ainsi, en Asie, la kétamine est l'une des principales substances détournées. L'utilisation chronique de kétamine, d'origine pharmaceutique ou de fabrication clandestine, est associée au développement de cystites à répétition, d'une incontinence urinaire et de douleurs lombaires chroniques [10].

Il existerait une toxicité directe de la kétamine et de ses métabolites à haute concentration sur les cellules interstitielles de la vessie, entraînant une inflammation chronique sous-muqueuse qui serait un facteur prédisposant aux infections urinaires basses, aux sténoses urétérales, et à une rétention urinaire chronique avec hydronéphrose. Des cas de nécrose papillaire ont également été décrits [11]. La kétamine est aussi un activateur du système sympathique, ce qui pourrait favoriser l'incontinence urinaire.

4.2.2. Insuffisance rénale aiguë pré-rénale : cannabis, cannabinoïdes de synthèse, amphétamines et dérivés, cathinones

Les insuffisances rénales aiguës (IRA) fonctionnelles sont retrouvées principalement dans le cadre de consommations de cannabis et de substances cannabinoïdes de synthèse, et sont associées à un syndrome d'hyperémèse [12]. Ce syndrome, découvert depuis le début des années 2000, se manifeste par des vomissements incoercibles et des douleurs abdominales. Il survient en période de consommation de cannabinoïdes et ne doit 


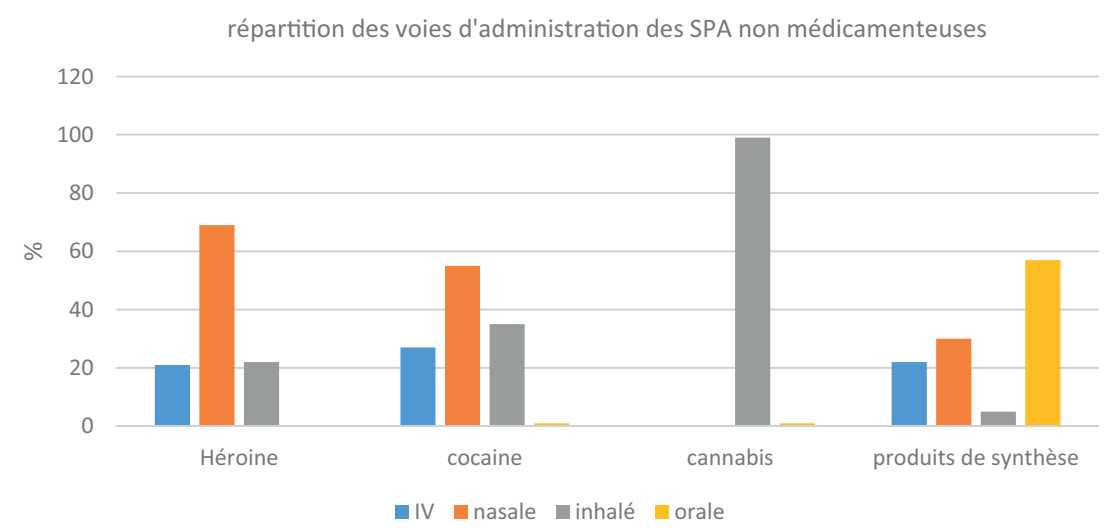

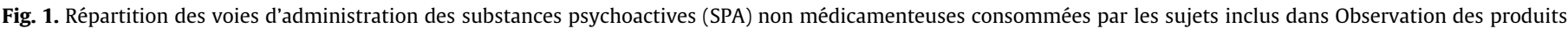

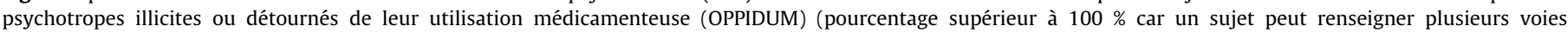
d'administration). IV : intraveineuse.

pas être confondu avec un syndrome de sevrage. L'IRA fonctionnelle survenant dans ce contexte est multifactorielle, liée à des pertes abondantes lors des vomissements, à une carence d'apports, et à des pertes hydriques cutanées, les douleurs étant calmées par les douches chaudes prises de façon pluriquotidienne par les patients en crise. Par ailleurs, la consommation de substances entraînant une élévation de la température corporelle par activation du système sérotoninergique, telles que les amphétamines et les cathinones, peut également engendrer des pertes hydriques et favoriser la survenue d'épisodes d'IRA fonctionnelle [13].

Enfin, les patients peuvent présenter un syndrome hépatorénal dans le cadre de consommation d'ecstasy, d'élimination hépatique et responsable d'hépatite aiguë grave [14].

4.2.3. Rhabdomyolyse et tubulopathies : cocaine, héroine, amphétamines et dérivés, médicaments de substitution, cannabinoides de synthèse, cathinones

La rhabdomyolyse est une complication classique chez les consommateurs de SPA. La libération importante de myoglobine peut aboutir à un dépassement des capacités de réabsorption tubulaire et à une nécrose tubulaire aiguë (NTA) $[15,16]$. Chez les consommateurs de SPA, plusieurs mécanismes ont été identifiés :

- l'activation du système sympathique, qui entraîne une vasoconstriction et un vasospasme des artérioles musculaires, aboutissant à une ischémie et une nécrose des cellules musculaires ;

- l'implication de facteurs traumatiques tels que des efforts extrêmes liés à l'absence de ressenti de la fatigue (rave party, par exemple) ou à des convulsions ;

- l'hyperthermie, secondaire à la prise de toxique et exacerbée par l'environnement extérieur où se trouve le consommateur, qui peut favoriser l'hypovolémie ;

- une toxicité directe de la cocaïne sur les cellules musculaires striées.

Les tableaux de rhabdomyolyse observés chez les consommateurs de SPA peuvent être particulièrement sévères [17]. Si elle en est le mécanisme le plus fréquent, la rhabdomyolyse n'est cependant pas seule responsable de NTA chez les utilisateurs de SPA.

Dans une étude de 2012, trois cas de NTA ont été identifiés chez 4 patients ayant présenté une IRA consécutive à la consommation de cannabinoïdes de synthèse, sans rhabdomyolyse. Des cristaux d'oxalate de calcium ont été retrouvés sur deux biopsies rénales, attribués aux métabolites issus des cannabinoïdes de synthèse [18].

De même, des cas de NTA ont été décrits après consommation de XLR-11 et UR-144. Ces deux cannabinoïdes de synthèse se fixent au récepteur cannabinoïde de type 1 (CB1), présent au niveau des podocytes et des cellules tubulaires proximales, dont l'activation pourrait avoir un rôle délétère [19].

Plusieurs cas de NTA et de tubulopathies proximales ont également été décrits chez des patients consommateurs de méthamphétamine [20], sans rhabdomyolyse.

L'héroïne, qui peut cristalliser à pH alcalin, peut entraîner des tubulopathies cristallines [21,22].

Enfin, les agents de coupages des SPA peuvent être des métaux lourds responsables de NTA ou de néphropathies tubulointerstitielles.

4.2.4. Néphropathies interstitielles : cocaïne, ecstasy, cannabinoides de synthèse

Dans le cadre de la consommation d'ecstasy, des cas de néphrite interstitielle aiguë (NIA) ont été décrits. La biopsie retrouvait une infiltration lymphocytaire prédominante. L'IRA était régressive spontanément [16].

Dans le cadre de la consommation de cocaïne, une NIA aiguë a été rapportée chez cinq patients afro-américains [23-27] présentant un infiltrat lymphocytaire et éosinophile ainsi qu'une réponse modérée aux corticoïdes.

Enfin, trois cas de NIA secondaire à la prise de cannabinoïdes de synthèse ont été rapportés [18,28,29], dont deux retrouvaient des cristaux d'oxalate de calcium pouvant être secondaires à des plantes utilisées en agent de coupage [30].

\subsubsection{Atteinte vasculaire : cocaine, amphétamines et dérivés, cannabis}

La cocaïne et les amphétamines peuvent être responsables de crise aiguë hypertensive voire d'hypertension artérielle (HTA) maligne avec microangiopathie thrombotique (MAT) [31]. Le mécanisme est un blocage de la recapture des catécholamines (dopamine, épinéphrine, norépinéphrine) entraînant une tachycardie et une HTA. De plus, ces SPA pourraient agir directement sur la contraction des cellules musculaires lisses via une augmentation de l'influx de calcium, jouant un rôle primordial dans la contraction de la cellule et le tonus vasculaire [16]. La cocaïne favorise par ailleurs la synthèse par les cellules musculaires lisses de récepteurs de l'endothéline 1, qui est un puissant vasoconstricteur [32].

Des cas d'infarctus rénaux ont été décrits après consommation de cocaïne, d'amphétamines et de cannabis [33-35]. Les mécanismes impliqués sont non seulement la vasoconstriction 
Tableau 3

Principales atteintes uro-néphrologiques liées aux substances psychoactives utilisées par les usagers de drogues.

\begin{tabular}{|c|c|c|c|c|c|c|c|c|c|c|}
\hline & \multicolumn{2}{|l|}{ Opioïdes } & \multicolumn{2}{|l|}{ Amphétamines } & \multirow{2}{*}{$\frac{\text { Anesthésiques }}{\text { Kétamine }}$} & \multicolumn{2}{|c|}{ Cannabinoïdes } & \multicolumn{3}{|c|}{ Médicaments de substitution } \\
\hline & Cocaïne & Héroüne & Ecstasy & Métamphétamines & & Cannabis & $\begin{array}{l}\text { Cannabinoïdes } \\
\text { de synthèse }\end{array}$ & Méthadone & $\begin{array}{l}\text { Buprénorphine } \\
\text { haut dosage }\end{array}$ & Cathinones \\
\hline Rhabdomyolyse & $\begin{array}{l}X \\
{[15-17]}\end{array}$ & $\begin{array}{l}X \\
{[15,16]}\end{array}$ & $\begin{array}{l}X \\
{[15,16]}\end{array}$ & $\begin{array}{l}X \\
{[15,16]}\end{array}$ & & & & $\begin{array}{l}X \\
{[15,16]}\end{array}$ & $\begin{array}{l}X \\
{[15,16]}\end{array}$ & $\begin{array}{l}X \\
{[15,16]}\end{array}$ \\
\hline Pré-rénale & & & $\begin{array}{l}X \\
{[13,14]}\end{array}$ & $\begin{array}{l}X \\
{[13]}\end{array}$ & & $\begin{array}{l}X \\
{[12]}\end{array}$ & $\begin{array}{l}X \\
{[12]}\end{array}$ & & & $\begin{array}{l}X \\
{[13]}\end{array}$ \\
\hline Vascularite & $\begin{array}{l}\mathrm{X} \\
{[40-42]}\end{array}$ & & $\begin{array}{l}X \text { (nécrosante) } \\
{[43,44]}\end{array}$ & $\begin{array}{l}X \\
{[44]}\end{array}$ & & & & & & \\
\hline $\begin{array}{l}\text { Néphrite } \\
\text { interstitielle }\end{array}$ & $\begin{array}{l}X \text { (aiguë) } \\
{[24-28]}\end{array}$ & & $\begin{array}{l}\mathrm{X} \text { (aiguë) } \\
{[17]}\end{array}$ & & & & $\begin{array}{l}\text { X (aiguë) } \\
{[19,29,30]}\end{array}$ & & & \\
\hline NTA & $\begin{array}{l}\mathrm{X} \\
\mathrm{X} \\
{[15,16]}\end{array}$ & & $\begin{array}{l}\mathrm{X} \\
{[21]}\end{array}$ & $\begin{array}{l}X \\
{[21]}\end{array}$ & & & $\begin{array}{l}X \\
{[19,20]}\end{array}$ & & & $\begin{array}{l}X \\
{[15,16]}\end{array}$ \\
\hline MAT/PAN & $\begin{array}{l}\mathrm{X}(\mathrm{MAT}) \\
{[31]}\end{array}$ & & $\begin{array}{l}\mathrm{X}(\mathrm{PAN}) \\
{[31]}\end{array}$ & & & & & & & \\
\hline $\begin{array}{l}\text { Hypertension } \\
\text { artérielle } \\
\text { (poussée ou } \\
\text { maligne) }\end{array}$ & $\begin{array}{l}X \\
{[31,33]}\end{array}$ & & $\begin{array}{l}X \\
{[31]}\end{array}$ & $\begin{array}{l}\text { X (papillaire) } \\
{[31]}\end{array}$ & & & & & & \\
\hline $\begin{array}{l}\text { Infarctus rénal, } \\
\text { nécrose }\end{array}$ & $X$ (corticale) & & $\begin{array}{l}\mathrm{X} \text { (vascularite) } \\
{[34-36]}\end{array}$ & & X (nécrose papillaire) & $\begin{array}{l}x \\
{[33-35]}\end{array}$ & & & & \\
\hline Thrombose & $\begin{array}{l}\mathrm{X} \text { (artérielle) } \\
{[37]}\end{array}$ & & $\begin{array}{l}\mathrm{X} \text { (veineuse et artérielle) } \\
{[38,39]}\end{array}$ & & & & & & & \\
\hline Tubulopathie & $\mathrm{X}$ (pigments) & $\begin{array}{l}\text { X (cristaux d'héroïne) } \\
{[22,23]}\end{array}$ & $\begin{array}{l}X \\
{[22]}\end{array}$ & & & & $\begin{array}{l}\text { X (oxalate de } \mathrm{Ca} 2+\text { ) } \\
{[19,31]}\end{array}$ & & & \\
\hline $\mathrm{HSF}$ & $\begin{array}{l}X \\
{[17]}\end{array}$ & $\begin{array}{l}\text { X (afro-américain, héroïne black) } \\
{[45,46]}\end{array}$ & & & & & $\begin{array}{l}\mathrm{X} \\
{[17]}\end{array}$ & & & \\
\hline GNMP & $\begin{array}{l}\mathrm{X} \\
{[51]}\end{array}$ & $\begin{array}{l}\text { X (caucasie, héroïne white) } \\
{[21]}\end{array}$ & $\begin{array}{l}\mathrm{X} \\
{[51]}\end{array}$ & $\begin{array}{l}X \\
{[51]}\end{array}$ & & $\begin{array}{l}X \\
{[49]}\end{array}$ & & & & \\
\hline GNA & & $\begin{array}{l}\text { X (post-endocardite) } \\
{[47]}\end{array}$ & & & & & & & & \\
\hline Amylose & & $\begin{array}{l}X \\
{[47]}\end{array}$ & & $\begin{array}{l}X \\
{[47]}\end{array}$ & & & & & & \\
\hline Hydronéphrose & & & & & $\begin{array}{l}X \\
{[11]}\end{array}$ & & & & & \\
\hline $\begin{array}{l}\text { Rejet aigu en } \\
\text { post-greffe }\end{array}$ & & $\begin{array}{l}X \\
{[38]}\end{array}$ & & & & & & & & \\
\hline $\begin{array}{l}\text { Troubles hydro- } \\
\text { électrolytiques }\end{array}$ & $\begin{array}{l}\text { X (hyponatrémie) } \\
{[52]}\end{array}$ & & $\begin{array}{l}\text { X (hyponatrémie) } \\
{[52]}\end{array}$ & & & & $\begin{array}{l}\text { X } \\
\text { (hypophosphorémie) } \\
\text { [53] }\end{array}$ & & & \\
\hline
\end{tabular}

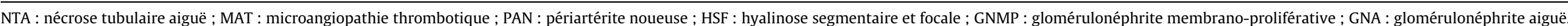


(inhibition de la recapture des catécholamines), mais également l'augmentation de l'agrégation plaquettaire et la synthèse accrue de thromboxane favorisant la thrombose. Des cas de thrombose aortique, de dissection ou de thrombose des artères rénales en lien avec la consommation de cocaïne ont été rapportés [36]. Des cas de thrombose veineuse rénale bilatérale ont également été décrits chez des consommateurs d'ecstasy, sans thrombose visible au Doppler mais avec confirmation de la présence de microthrombi après biopsie, le plus souvent dans un contexte avéré de coagulation intravasculaire disséminée [37].

Enfin, la consommation d'ecstasy chez les donneurs de rein a été associée à des cas d'IRA post-greffe chez le receveur. C'est notamment le cas d'une donneuse décédée dont les deux receveurs ont développé une IRA anurique vers j7. L'analyse anatomopathologique post-transplantectomie retrouvait des zones de nécrose parenchymateuse, avec des thromboses vasculaires (notamment artérielles) et de la nécrose fibrinoïde. Cette nécrose avait été attribuée à la consommation d'ecstasy par la donneuse décédée [38].

\subsubsection{Vascularite : lévamisole/cocaine, amphétamines et dérivés}

La cocaïne peut contenir un agent de coupage nommé lévamisole, qui peut être responsable d'une vascularite nécrosante mimant une vascularite à ANCA. Les premiers cas ont été décrits dans les années 1970 avec une atteinte initiale articulaire et cutanée à type de nécrose des extrémités, du nez et/ou des oreilles. Des atteintes rénales ont été décrites depuis 2010. Le lévamisole est un antagoniste du récepteur nicotinique à l'acétylcholine, qui était utilisé comme anti-helminte en médecine vétérinaire. Il a aussi été employé comme immunosuppresseur dans le cadre du traitement de glomérulopathies, de la polyarthrite rhumatoïde ou du cancer colorectal. Retiré du marché dans de nombreux pays, il reste testé comme traitement de l'anémie post-aplasie [39].

Le lévamisole peut se comporter comme une haptène, entraînant la formation d'auto-anticorps. On peut ainsi retrouver chez ces patients des anticorps anti-cytoplasme des polynucléaires neutrophiles (ANCA), avec une possible spécificité anti-protéinase 3 ou anti-myélopéroxidase [40], des anticorps anti-nucléaires, et des anti-cardiolipine [39]. La présence d'ANCA dirigés contre l'élastase des neutrophiles (anti-HNE) a également été rapportée et plaide en faveur d'une imputabilité de la consommation de lévamisole/cocaïne. Ainsi, des anti-HNE ont été retrouvés chez 14 patients sur 25 présentant une vascularite associée à la cocaïne, contre 3 patients sur 604 présentant une vascularite non liée au lévamisole/cocaïne [41].

Par ailleurs, le lévamisole aurait un effet synergique avec la cocaïne sur l'activation du récepteur nicotinique à l'acétylcholine, accentuant la vasoconstriction [40]. La biopsie rénale, lorsqu'elle est réalisée, peut montrer une prolifération mésangiale, des lésions inflammatoires vasculaires et des microthrombi.

Un cas de vascularite nécrosante a également été décrit chez un patient ayant eu une consommation récréative d'ecstasy 10 jours auparavant, sans antécédent de toxicomanie [42]. De même, la consommation récente d'amphétamines était le seul facteur commun retrouvé dans une cohorte de 1970 regroupant 14 patients multi-toxicomanes présentant un tableau de périartérite noueuse (vascularite nécrosantes et micro-anévrismes) [43].

\subsubsection{Hyalinose segmentaire et focale, et glomérulosclérose : héroine,} cocaine

La consommation d'héroïne est associée au développement d'une hyalinose segmentaire et focale (HSF). L'HSF représente en effet la principale cause d'IRA chez ces patients, avec notamment 11 HSF retrouvées sur 13 biopsies réalisées chez des patients afroaméricains consommateurs d'héroïne en 1974 [44]. Cette atteinte peut évoluer vers une sclérose glomérulaire complète.
De même, une étude de 1980 regroupant 21 patients ayant eu une biopsie pour insuffisance rénale montrait une glomérulosclérose (de segmentaire et focale à globale et diffuse) chez 11 patients, et une sclérose glomérulaire totale chez 8 patients [45]. Dans cette étude, le risque de sclérose était augmenté de plus de 40 fois chez les consommateurs d'héroïne par rapport aux non-consommateurs. Sur toutes les biopsies, des dépôts non spécifiques d'IgM, IgG et C3 étaient retrouvés.

Le risque d'HSF et de glomérulosclérose globale est majoré dans la population noire et lors de consommation d'héroïne black tar, produite au Mexique et encore peu développée en France, dont le procédé de transformation plus rapide conduit à la présence de plus d'impuretés.

Plusieurs mécanismes sont impliqués :

- un élargissement mésangial secondaire à une inhibition de la dégradation de la matrice extracellulaire par activation des inhibiteurs de métalloprotéases ;

- une altération des cellules épithéliales glomérulaires secondaire au stress oxydant, par toxicité directe [32] aboutissant à une sclérose glomérulaire ;

- l'activation du système rénine-angiotensine, par l'augmentation de la production de TGF bêta, favorisant la fibrogenèse [32].

Par ailleurs, l'héroïne pourrait favoriser l'apoptose des cellules épithéliales glomérulaires, par analogie à la morphine dont elle est le dérivé.

Une étude de 1997, comparant les lésions histologiques rénales de 40 personnes décédées après consommation de cocaïne à celles de 40 personnes décédées après accident de la route, a montré une incidence plus élevée de lésions de HSF et d'athérosclérose dans le groupe cocaïne [17].

Il est important de souligner que ces patients sont plus souvent infectés par le VIH, rendant difficile la distinction entre la part toxique et la part virale sur ces lésions de HSF.

\subsubsection{Autres glomérulopathies}

Les consommateurs de SPA par voie intraveineuse peuvent présenter une glomérulonéphrite aiguë post-infectieuse consécutive à un abcès cutané ou une endocardite infectieuse [46]. L'inflammation chronique secondaire à des infections cutanées à répétition peut également conduire au développement d'une amyloses AA, en particulier chez les injecteurs de SPA par voie sous-cutanée recherchant une absorption plus lente du produit et un effet prolongé, mais au prix d'une stase sous-cutanée avec risque accru d'infection [47]. Des cas de glomérulonéphrite extra-membraneuse (GEM) ont été rapportées chez des patients consommateurs d'héroïne ou de cannabis [48].

Par ailleurs, des glomérulonéphrites membrano-prolifératives (GNMP) ont été décrites après consommation de cannabis [49], de cocaïne [50], d'héroïne [21] et d'amphétamine [51].

\subsubsection{Sur-risque d'insuffisance rénale chronique}

Les atteintes rénales secondaires aux SPA sont, pour la majeure partie, réversibles avec cependant parfois nécessité de dialyse temporaire. Cependant, certaines atteintes peuvent conduire à une insuffisance rénale chronique (IRC), notamment en cas d'HSF/ glomérulosclérose ou de lésions vasculaires d'athérosclérose [33]. La consommation de cocaïne serait ainsi associée à un risque multiplié par 3 d'IRC [52], qui pourrait encore accentuer leur surrisque cardiovasculaire.

\subsubsection{Désordres hydro-électrolytiques : cocaine, ecstasy}

L'ecstasy et la cocaïne stimulent la sécrétion de vasopressine. Celle-ci peut être responsable d'une hyponatrémie avec tableau de sécrétion inappropriée d'hormones antidiurétiques (SIADH). 


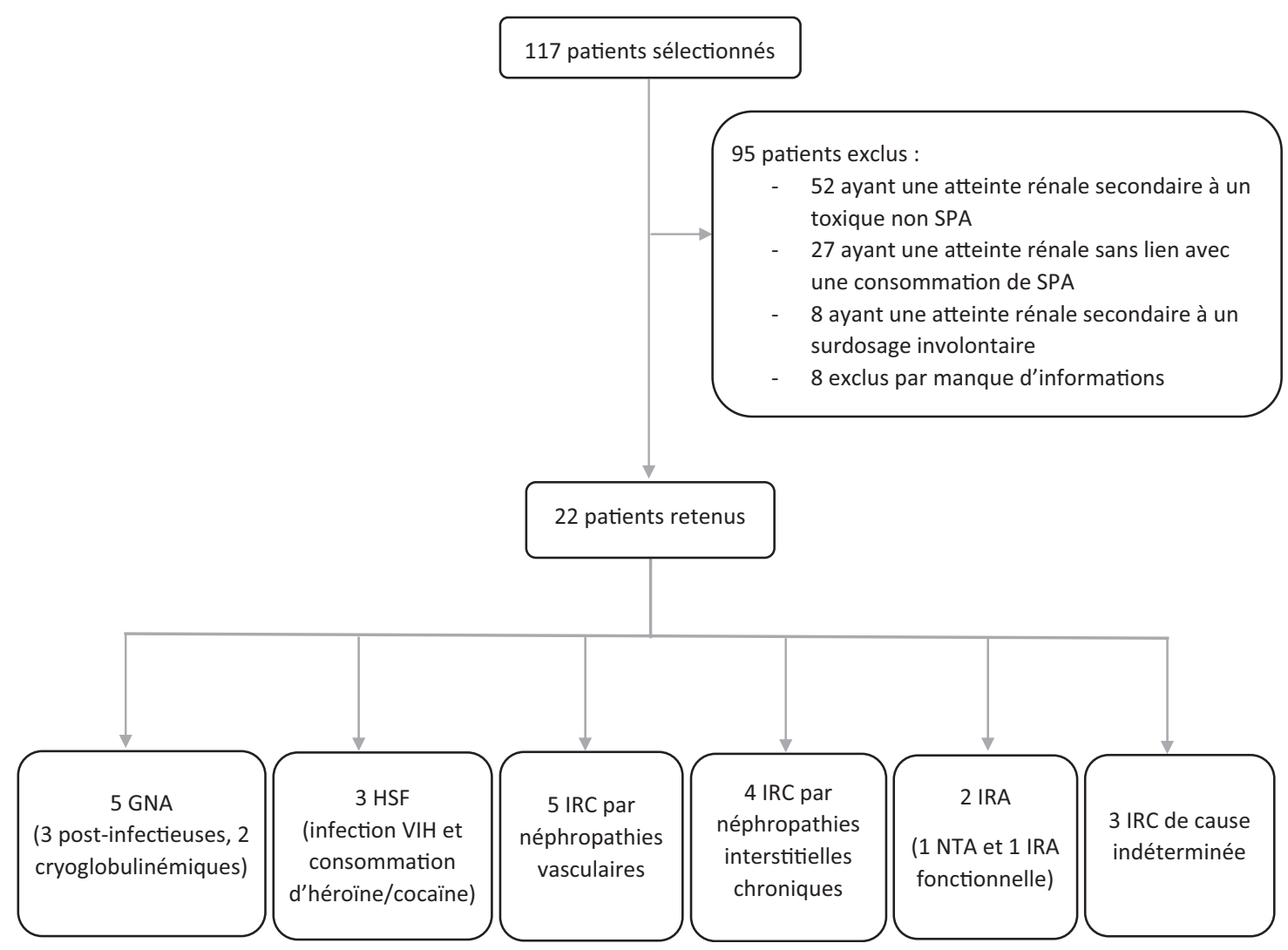

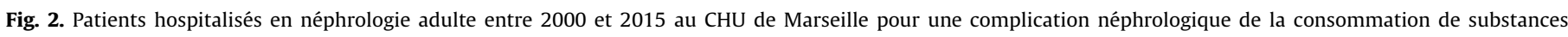

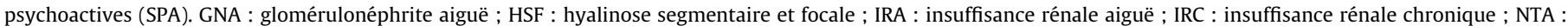
nécrose tubulaire aiguë.

Différents facteurs peuvent exacerber ce phénomène, notamment l'hypovolémie secondaire à l'hyperthermie, et les boissons abondantes en réponse à une sensation de soif accrue.

Le lévamisole, associé à la cocaïne dans 70 \% des cas environ, peut également entraîner des hyponatrémies par SIADH ou perte de sel d'origine tubulaire rénale [52].

Récemment, une série de cas d'hypophosphorémie a été rapportée dans le cadre de syndrome d'hyperémèse chez les patients consommateurs de cannabinoïdes de synthèse [53].

\subsection{Enquête sur les hospitalisations en néphrologie au CHU de Marseille}

Parmi les 16030 séjours en hospitalisation en néphrologie adulte au CHU de Marseille de janvier 2000 à décembre 2015, 117 patients ont été sélectionnés à partir des codes diagnostiques issus du PMSI et de la prescription de TSO. Après analyse de chaque dossier, nous avons identifié 22 patients hospitalisés pour une complication néphrologique liée à la consommation d'une SPA non médicamenteuse (soit 0,14 \% des patients hospitalisés en néphrologie) (Fig. 2).

Il s'agissait de 19 (86\%) hommes et 3 (14\%) femmes, d'un âge médian de 47 ans ( 18 à 57 ans). La répartition de ces cas au cours du temps est représentée sur la Fig. 3. L'année 2013 est marquée par un pic de fréquence des hospitalisations pour complications néphrologiques des SPA ( 7 cas annuels contre 1 à 3 cas pour les autres années), sans qu'une substance ou un mode d'administration commun n'ait été mis en évidence.

Les caractéristiques des patients et les complications observées sont résumées dans le Tableau 4.

Cinq patients ont présenté une glomérulonéphrite aiguë : 3 GNA au cours d'endocardites infectieuses, chez un consommateur de cocaïne par voie intraveineuse (IV) et 2 consommateurs d'héroïne IV, et 2 glomérulonéphrites cryoglobulinémiques chez des consommateurs d'héroïne IV dont l'un était coinfecté VIH/VHC.

Trois cas d'HSF ont été documentés par biopsie rénale, chez 2 patients d'origine africaine consommateurs d'héroïne IV et de cocaïne, infectés par le VIH et non traités, et chez 1 patient caucasien consommateur de cocaïne sniffée, infecté par le VIH et en rupture de trithérapie antirétrovirale depuis 2 ans. Ces 3 cas s'accompagnaient d'une dégradation rapide de la fonction rénale, ayant conduit les 2 premiers patients en dialyse, alors que le $3^{\mathrm{e}}$ stabilisait sa fonction rénale après contrôle tensionnel et reprise de la trithérapie.

Cinq patients présentaient une IRC modérée à sévère liée à une néphropathie vasculaire documentée par biopsie rénale, dont 4 étaient consommateurs d'héroïne et de cocaïne, et 1 de cannabis.

Quatre cas de néphropathie interstitielle chronique ont été mis en évidence chez 3 patients consommant de multiples SPA, et une patiente consommant exclusivement de l'ecstasy et ayant présenté une hépatite fulminante ayant nécessité une transplantation hépatique en urgence, suivie d'une transplantation rénale.

Deux patients ont par ailleurs présenté une IRA, soit fonctionnelle chez un consommateur de SPA multiples (cocaïne, LSD, cannabis, héroïne), soit par NTA chez un consommateur de cocaïne.

Enfin, l'IRC a été découverte à un stade évolué chez trois patients consommateurs de cannabis et/ou de cocaïne, sans autre orientation étiologique.

\section{Discussion et conclusion}

Il existe une grande diversité des SPA consommées en France. Les complications somatiques liées à l'usage de SPA sont 


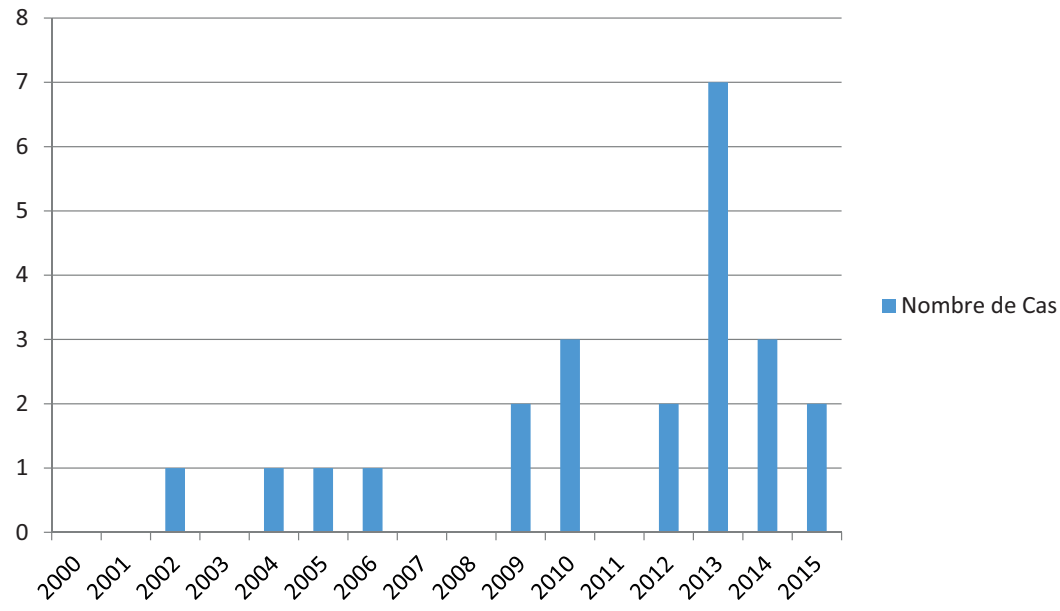

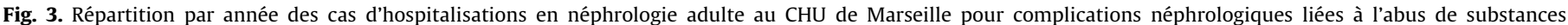
psychoactives, de 2000 à 2015. Le pic observé en 2013 n'a pu être relié à un produit ou un mode de consommation commun.

Tableau 4

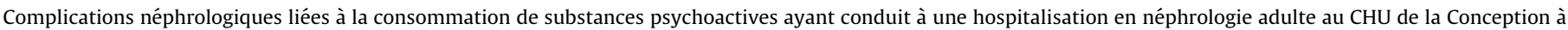
Marseille, de 2000 à 2015.

\begin{tabular}{|c|c|c|c|c|}
\hline Genre et âge & Année & Atteinte rénale & SPA & Co-infections \\
\hline Homme, 48 ans & 2013 & GNA post-endocardite infectieuse & Cocaïne & \\
\hline Homme, 40 ans & 2013 & GNA post-endocardite infectieuse & Héroïne, buprénorphine & $\mathrm{VHC}+$ \\
\hline Homme, 48 ans & 2013 & GNA post-endocardite infectieuse & Héroïne, buprénorphine & $\mathrm{VHB}+, \mathrm{VHC}+$ active \\
\hline Homme, 52 ans & 2013 & Cryoglobulinémie et néphro-angiosclérose & Héroïne, buprénorphine & $\mathrm{VIH}+, \mathrm{VHC}+$ \\
\hline Femme, 35 ans & 2009 & Cryoglobulinémie & Héroïne, buprénorphine & \\
\hline Homme, 36 ans & 2009 & Néphropathie du VIH (HSF) & Cocaïne, LSD, héroïne & $\mathrm{VIH}+$ \\
\hline Homme, 36 ans & 2004 & Néphropathie du VIH (HSF) & Héroïne & VIH+ \\
\hline Femme, 42 ans & 2013 & Néphropathie vasculaire & Héroïne, buprénorphine & \\
\hline Homme, 48 ans & 2014 & Néphropathie vasculaire, fibrose & Héroïne, buprénorphine & $\mathrm{VIH}+, \mathrm{VHC}+$ \\
\hline Homme, 49 ans & 2012 & Néphropathie vasculaire & Héroïne, cocaïne & $\mathrm{VIH}+, \mathrm{VHC}+$ (non traitée) \\
\hline Homme, 48 ans & 2013 & Néphropathie vasculaire & Héroïne, méthadone & $\mathrm{VIH}+, \mathrm{VHC}+$ et $\mathrm{VHB}+$ \\
\hline Homme, 43 ans & 2005 & Néphropathie vasculaire & Cannabis & \\
\hline Homme, 48 ans & 2014 & $\mathrm{~N}$ chronique & Héroïne, buprénorphine & VHC+ active \\
\hline Femme, 18 ans & 2015 & NTIC & Ecstasy & \\
\hline Homme, 50 ans & 2015 & NTIC & Héroïne, buprénorphine & $\mathrm{VIH}+, \mathrm{VHB}+, \mathrm{VHC}+$ \\
\hline Homme, 32 ans & 2002 & NTIC & Héroïne, cannabis, buprénorphine & \\
\hline Homme, 29 ans & 2010 & IRA fonctionnelle & Héroïne, cocaïne, cannabis, LSD & \\
\hline Homme, 48 ans & 2013 & IRA (NTA) & Héroïne, cannabis, buprénorphine & VHC+ \\
\hline Homme, 57 ans & 2012 & Néphropathie indéterminée, fibrose & Cocaïne & \\
\hline Homme, 46 ans & 2006 & Néphropathie indéterminée & Cannabis & VHC + active (non traitée) \\
\hline Homme, 42 ans & 2014 & Néphropathie indéterminée & Intoxication aux morphiniques & $\mathrm{VHC}+$ \\
\hline
\end{tabular}

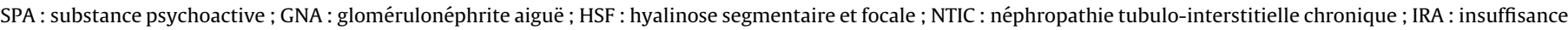
rénale aiguë ; VIH : virus de l'immunodéficience humaine ; VHB : virus de l'hépatite B ; VHC : virus de l'hépatite C.

probablement sous-estimées : mal connues par les usagers et les médecins, elles peuvent être plus difficilement repérées, et sousnotifiées. L'imputabilité spécifique d'une SPA est difficile à établir en cas de consommation multiple ou chez les patients présentant des infections virales chroniques. Les atteintes uro-néphrologiques liées à l'abus de SPA sont variées, et la consommation de telles substances, même festive et occasionnelle, doit être recherchée en néphrologie de façon plus systématique à l'interrogatoire, au même titre que la consommation de médicaments tels que les antiinflammatoires. Pour chaque SPA consommée, il est utile de caractériser l'ancienneté de la prise, les modalités de consommation et les effets recherchés. L'apport et la collaboration avec les pharmacologues du Centre d'addictovigilance dans cette évaluation est importante pour aider au diagnostic de symptômes liés à la consommation de SPA. Dans le doute et en l'absence d'autre étiologie retrouvée, on peut recourir à des analyses toxicologiques pour rechercher la présence de SPA. Les analyses immunochimiques permettent de dépister sur un prélèvement urinaire recueilli au plus tôt la présence des principales classes de produits (benzodiazépines, opiacés, cannabis, amphétamines, cocaïne, buprénorphine, méthadone...). D’autres SPA ne sont décelées que par des méthodes plus performantes telles que la chromatographie gazeuse couplée à la spectrométrie de masse sur le sang et/ ou sur les urines, réalisées dans les laboratoires spécialisés de pharmacologie/toxicologie des CHU. Il est également possible de faire analyser un échantillon de produit par le Système d'identification national des toxiques et substances (SINTES) qui répertorie, à des fins de santé publique, les substances circulant en France.

La déclaration des complications sanitaires liées à l'usage de SPA au réseau français d'addictovigilance, au même titre que pour la pharmacovigilance, est essentielle pour :

- évaluer la prévalence, améliorer le dépistage et la prévention de ces complications ;

- donner l'alerte sur la toxicité particulière de nouveaux produits, de nouveaux produits de coupe (par exemple lévamisole) ou de nouveaux usages ; 
- permettre des actions d'information des professionnels de santé et des usagers.

Les fiches de notification sont disponibles sur le site de l'ANSM : http://ansm.sante.fr/Declarer-un-effet-indesirable/ Pharmacodependance-Addictovigilance/Adresses-des-CEIP/ (offset)/3. Le faible nombre de cas de complications néphrologiques des SPA ayant conduit à une hospitalisation au CHU de Marseille reflète probablement un défaut d'interrogatoire (lié en partie à la méconnaissance par les médecins des SPA existantes et de leurs effets potentiels) et/ou un biais de codage. Cette enquête a permis de déclarer ces cas au centre d'addictovigilance, ce qui n'avait pas été fait jusqu'alors, et d'initier une collaboration entre néphrologues et addictovigilants.

\section{Déclaration de liens d'intérêts}

Les auteurs déclarent ne pas avoir de liens d'intérêts.

\section{Références}

[1] Peyrière H, Eiden C, Mallaret M, Victorri-Vigneau C. Medical complications of psychoactive substances with abuse risks: detection and assessment by the network of French addictovigilance centres. Therapie 2016;71:563-73.

[2] Jouanjus E, Lapeyre-Mestre M, Micallef J, The French Association of the Regional Abuse and Dependence Monitoring Centres. Cannabis use: signal of increasing risk of serious cardiovascular disorders. J Am Heart Assoc 2014;3:e000638

[3] Ibrahim S, Al-Saffar F, Wannenburg T. A unique case of cardiac arrest following K2 abuse. Case Rep Cardiol 2014;2014:120607.

[4] McKeever RG, Vearrier D, Jacobs D, LaSala G, Okaneku J, Greenberg MI. K2-Not the spice of life: synthetic cannabinoids and ST elevation myocardial infarction: a case report. J Med Toxicol 2015;11:129-31.

[5] Stankowski RV, Kloner RA, Rezkalla SH. Cardiovascular consequences of cocaine use. Trends Cardiovasc Med 2015;25:517-26.

[6] Lavender TW, McCarron B. Acute infections in intravenous drug users. Clin Med 2013;13:511-3.

[7] Chavant F, Boucher A, Le Boisselier R, Deheul S, Debruyne D. New synthetic drugs in addictovigilance. Therapie 2015;70:167-89.

[8] Luciano R, Perazella MA. Nephrotoxic effects of designer drugs: synthetic is not better! Nat Rev Nephrol 2014;10:314-24.

[9] Bryden AA, Rothwell PJ, O'Reilly PH. Urinary retention with misuse of "ecstasy". BMJ 1995;310:504.

[10] Pappachan JM, Raj B, Thomas S, Hanna FW. Multiorgan dysfunction related to chronic ketamine abuse. Proc (Bayl Univ Med Cent) 2014;27:223-5.

[11] Lee PY, Ong TA, Chua CB, Lei CCM, Teh GC. Street ketamine-associated bladder dysfunction: an emerging health problem. Malays Fam Phys 2009;4:15-8.

[12] Habboushe J, Sedor J. Cannabinoid hyperemesis acute renal failure: a common sequela of cannabinoid hyperemesis syndrome. Am J Emerg Med 2014; 32:690.

[13] Asser A, Taba P. Psychostimulants and movement disorders. Front Neurol 2015;6:75

[14] Ellis AJ, Wendon JA, Portmann B, Williams R. Acute liver damage and ecstasy ingestion. Gut 1996;38:454-8.

[15] Pendergraft WF, Herlitz LC, Thornley-Brown D, Rosner M, Niles JL. Nephrotoxic effects of common and emerging drugs of abuse. Clin J Am Soc Nephrol 2014;9:1996-2005.

[16] Berney-Meyer L, Putt T, Schollum J, Walker R. Nephrotoxicity of recreational party drugs. Nephrology (Carlton) 2012;17:99-103.

[17] Kosmadakis G, Michail O, Filiopoulos V, Papadopoulou P, Michail S. Acute kidney injury due to rhabdomyolysis in narcotic drug users. Int J Artif Organs 2011;34:584-8.

[18] Bhanushali GK, Jain G, Fatima H, Leisch LJ, Thornley-Brown D. AKI associated with synthetic cannabinoids: a case series. Clin J Am Soc Nephrol 2013;8: 523-6.

[19] Srisung W, Jamal F, Prabhakar S. Synthetic cannabinoids and acute kidney injury. Proc (Bayl Univ Med Cent) 2015;28:475-7.

[20] Kwon C, Zaritsky A, Dharnidharva VR. Transient proximal tubular renal injury following ecstasy ingestion. Pediatr Nephrol 2003;18:820-2.

[21] Bautista JE, Merhi B, Gregory O, Hu S, Henriksen K, Gohh R. Heroin crystal nephropathy. Clin Kidney J 2015;8:339-42.

[22] Fulton CC, Dalton JB. Micro-crystal identification tests for morphine, heroin, dilaudid, and cocaines. J Crim Law Criminol 2014;32:358-65.

[23] Alvarez D, Nzerue CM, Daniel JF, Faruque S, Hewan-Lowe K. Acute interstitial nephritis induced by crack cocaine binge. Nephrol Dial Transplant 1999; $14: 1260-2$.
[24] Decelle L, Cosyns JP, Georges B, Jadoul M, Lefebvre C. Acute interstitial nephritis after cocaine sniffing. Clin Nephrol 2007;67:105-8.

[25] Wojciechowski D, Kallakury B, Nouri P. A case of cocaine-induced acute interstitial nephritis. Am J Kidney Dis 2008;2:204-9.

[26] Alfaro R, Vasavada N, Paueksakon P, Hernandez GT, Aronoff GR. Cocaineinduced acute interstitial nephritis: a case report and review of the literature. J Nephropathol 2013;2:204-9.

[27] Bahaa Aldeen M, Talibmamury N, Alalusi S, Nadham O, Omer AR, Smalligan RD. When coke is not hydrating: cocaine-induced acute interstitial nephritis. J Investig Med High Impact Case Rep 2014;2 [2324709614551557].

[28] Kirkey K, MacMaster K, Suryaprasad A, Xu F, Klevens M, Roberts H, et al. Acute kidney injury associated with synthetic cannabinoid use - Multiple states, 2012. MMWR Morb Mortal Wkly Rep 2013;62:93-8.

[29] Thornton SL, Wood C, Friesen MW, Gerona RR. Synthetic cannabinoid use associated with acute kidney injury. Clin Toxicol 2013;51:189-90.

[30] Kamel M, Thajudeen B. A case of acute kidney injury and calcium oxalate deposition associated with synthetic cannabinoids. Saudi J Kidney Dis Transpl 2015;26:802-3.

[31] Lamia R, El Ati Z, Ben Fatma L, Zouaghi K, Smaoui W, Rania K, et al. Malignant hypertension-associated thrombotic microangiopathy following cocaine use. Saudi J Kidney Dis Transpl 2016;27:153-6.

[32] Hendricks-Munoz KD, Gerrets RP, Higgins RD, Munoz JL, Caines VV. Cocainestimulated endothelin-1 release is decreased by angiotensin-converting enzyme inhibitors in cultured endothelial cells. Cardiovasc Res 1996;31:117-23.

[33] Le Guen PY, Gestin S, Plat E, Quéhé P, Bressollette L. Renal and spleen infarction after massive consumption of cannabis and cocaine in a young man. J Mal Vasc 2011;36:41-4.

[34] Bemanian S, Motallebi M, Nosrati SM. Cocaine-induced renal infarction: report of a case and review of the literature. BMC Nephrol 2005;6:10.

[35] Hoefsloot W, de Vries RA, Bruijnen R, Bosch FH. Renal infarction after cocaine abuse: a case report and review. Clin Nephrol 2009;72:234-6.

[36] Edmondson DA, Towne JB, Foley DW, Abu-Hajir M, Kochar MS. Cocaineinduced renal artery dissection and thrombosis leading to renal infarction. WMJ 2004;103:66-9.

[37] Eldehni MT, Roberts ISD, Naik R, Vaux E. Case report of ecstasy-induced renal venous thrombosis. NDT Plus 2010;3:459-60

[38] Hurault de Ligny B, El Haggan W, Comoz F, Lobbedez T, Pujo M, Griveau AM, et al. Early loss of two renal grafts obtained from the same donor: role of ecstasy? Transplantation 2005;80:153-6.

[39] Garg L, Gupta S, Swami A, Zhang P. Levamisole/cocaine-induced systemic vasculitis and immune complex glomerulonephritis. Case Rep Nephrol 2015;2015:372413.

[40] McGrath MM, Isakova T, Rennke HG, Mottola AM, Laliberte KA, Niles JL. Contaminated cocaine and antineutrophil cytoplasmic antibody-associated disease. Clin J Am Soc Nephrol 2011;6:2799-805.

[41] Wiesner O, Russell KA, Lee AS, Jenne DE, Trimarchi M, Gregorini G, et al. Antineutrophil cytoplasmic antibodies reacting with human neutrophil elastase as a diagnostic marker for cocaine-induced midline destructive lesions but not autoimmune vasculitis. Arthritis Rheum 2004;50:2954-65.

[42] Bingham C, Beaman M, Nicholls AJ, Anthony PP. Necrotizing renal vasculopathy resulting in chronic renal failure after ingestion of methamphetamine and 3,4-methylenedioxymethamphetamine ('ecstasy'). Nephrol Dial Transplant 1998;13:2654-5.

[43] Citron BP, Halpern M, McCarron M, Lundberg GD, McCormick R, Pincus IJ, et al. Necrotizing angiitis associated with drug abuse. N Engl J Med 1970;283: 1003-11.

[44] Rao TK, Nicastri AD, Friedman EA. Natural history of heroin-associated nephropathy. N Engl J Med 1974;290:19-23.

[45] Cunningha EE, Brentjens JR, Zielezny MA, Andres GA, Venuto RC. Heroin nephropathy: a clinicopathologic and epidemiologic study. Am J Med 1980;68:47-53.

[46] Boils CL, Nasr SH, Walker PD, Couser WG, Larsen CP. Update on endocarditisassociated glomerulonephritis. Kidney Int 2015;87:1241-9.

[47] Neugarten J, Gallo GR, Buxbaum J, Katz LA, Rubenstein J, Baldwin DS. Amyloidosis in subcutaneous heroin abusers ("skin poppers' amyloidosis"). Am J Med 1986;81:635-40.

[48] Bohatyrewicz M, Urasinska E, Rozanski J, Ciechanowski K. Membranous glomerulonephritis may be associated with heavy marijuana abuse. Transplant Proc 2007;39:3054-6.

[49] Peces R, Navascués RA, Baltar J, Seco M, Alvarez J. Antiglomerular basement membrane antibody-mediated glomerulonephritis after intranasal cocaine use. Nephron 1999:81:434-8.

[50] Jones ES, Rayner BL. Hypertension, end-stage renal disease and mesangiocapillary glomerulonephritis in methamphetamine users. S Afr Med J 2015;105:199-201.

[51] Vupputuri S, Batuman V, Muntner P, Bazzano LA, Lefante JJ, Whelton PK, et al. The risk for mild kidney function decline associated with illicit drug use among hypertensive men. Am J Kidney Dis 2004;43:629-35.

[52] Friend K, Milone MC, Perrone J. Hyponatremia associated with levamisoleadulterated cocaine use in emergency department patients. Am Coll Emerg Phys 2016;60:94-6.

[53] Cadman PE. Hypophosphatemia in users of cannabis. Am J Kidney Dis 2016;69:152-5. 